Cahiers de la recherche sur les droits

Cahiers

Fur les Droits fondamentaux

$11 \mid 2013$

Le droit de la famille en (r)évolutions

\title{
Le paramilitarisme et les résultats du processus de Justice et de Paix en Colombie
}

Didima Rico Chavarro et Karen Chadoutaud

\section{(2) OpenEdition}

Édition électronique

URL : https://journals.openedition.org/crdf/4685

DOI : $10.4000 /$ crdf.4685

ISSN : 2264-1246

Éditeur

Presses universitaires de Caen

Édition imprimée

Date de publication : 1 novembre 2013

Pagination : 131-145

ISSN : 1634-8842

\section{Référence électronique}

Didima Rico Chavarro et Karen Chadoutaud, «Le paramilitarisme et les résultats du processus de

Justice et de Paix en Colombie ", Cahiers de la recherche sur les droits fondamentaux [En ligne], 11 |

2013, mis en ligne le 01 décembre 2014, consulté le 14 novembre 2022. URL : http://

journals.openedition.org/crdf/4685; DOI : https://doi.org/10.4000/crdf.4685 


\title{
Le paramilitarisme et les résultats du processus de Justice et de Paix en Colombie ${ }^{1}$
}

\author{
Didima RICO CHAVARRO \\ Professeure de droit pénal et droits de l'homme à l'Université autonome de Colombie \\ Groupe de recherche "Libertad y Garantismo "
}

\section{Karen CHADOUTAUD}

Professeure en droits de l'homme à I'Université Incca de Colombie

Groupe de recherche "Libertad y Garantismo »

I. Le contexte de l'extension du paramilitarisme

II. La stratégie de constitution et de consolidation du modèle politique de guerre

III. Les progrès du processus de Justice et de Paix face au paramilitarisme

Annexe - Tableaux et analyses de l'application de la loi de Justice et de Paix cinq ans après sa mise en place

\section{Le contexte de l'extension du paramilitarisme}

Le phénomène social du paramilitarisme a changé de forme. À l'heure actuelle, il est nommé conventionnellement sous la forme linguistique de «bandas crimineles» (Bacrim) ou «successeurs des paramilitaires » ou «néoparamilitarisme». Ce changement sémantique est de toute évidence une stratégie, constituée par un ensemble de récits linguistiques promus de manière intentionnelle par le pouvoir qui souhaite que pour l'imaginaire collectif ce phénomène soit lu et interprété comme neuf, comme l'a indiqué Rodolfo Arango: «La guerre est menée non seulement sur le champ de bataille mais dans le langage, dont la gestion est essentielle pour la légitimité des acteurs et la confrontation ${ }^{3}$.

1. Article fondé sur le rapport final du travail de recherche préparé par le groupe «Libertad y Garantismo », auparavant dénommé «Estudios políticos, normativos y de derechos humanos» (Colciencias, catégorie D), formé par G. Cuartas, K. Chadoutaud, R. Vargas et M. Barreto. Traduction de l'espagnol effectuée par K. Chadoutaud.

2. Elizabeth Lira, in Memoria histórica y reparación emocional. Mujeres de África y América en lucha por la verdad, la justicia y la reparación, San Salvador, Las Dignas - Asociación de mujeres por la dignidad y la vida, janvier 200o, p. 24. Elizabeth Lira est une psychologue chilienne; elle a reçu le premier prix au IV e concours national d'essais «Premio anual profesor Jorge Millas", pour une culture de respect des droits de l'homme, avec la contribution: Y a los ojos se me asomará la vida que ya viví.

3. R. Arango, «Cinco años de justicia y paz, camino hacia la impunidad», Razón Pública (revue en ligne), 29 juin 2010 , disponible à l'adresse: http://www.razonpublica.com/index.php/politica-y-gobierno-temas-27/1210-cinco-anos-de-justicia-y-paz-camino-hacia-la-impunidad-.html. 
Les médias répandent l'idée selon laquelle le paramilitarisme a disparu avec la démobilisation et la réinsertion des paramilitaires, et qu'il y a eu des progrès dans le processus de paix. Mais devant l'augmentation inattendue du paramilitarisme dans toutes les régions du pays, exprimé par toutes sortes d'actes illégaux qui soumettent la société à la peur et participent au développement d'un sentiment d'impunité, on évite de soulever l'échec du processus ${ }^{4}$ et

[...] l'aggravation continue de la situation humanitaire et des droits humains, avec des preuves de violations graves et généralisées à l'encontre des civils y compris des violences sexuelles, massacres, exécutions extrajudiciaires, menaces et extorsions 5 .

Nous avons ainsi pu constater, malgré la diminution des violations systématiques des droits de l'homme, une présence toujours forte du conflit armé et de la dégradation qu'il opère, la persistance de facteurs structurels et une pauvreté qui demeure à des niveaux dévastateurs, malgré la réduction, d'après les statistiques, de $46 \%$ et de $17 \%$ de l'extrême pauvreté en $2008^{6}$. Les profondes inégalités sociales sont la cause fondamentale de l'état de guerre persistant que subit la Colombie depuis 1962.

La persistance de tous ces facteurs et le mécontentement social ont conduit à la formation de guérillas d'insurgés, qui se sont opposées aux institutions politiques pendant plusieurs décennies durant la seconde moitié du $\mathrm{XX}^{\mathrm{e}}$ siècle. Ces guérillas ont pris les armes en prétendant représenter la population exclue et marginalisée pour réclamer la justice sociale ${ }^{7}$. Bien que l'un des thèmes clés de tous les gouvernements ait été de mettre fin à la montée des guérillas en confiant aux forces armées la mission historique de les éliminer, les groupes de rebelles armés ont grandi et se sont renforcés au fil des ans.

C'est pourquoi, les institutions armées, dans le cadre des politiques étatiques, utilisent une stratégie militaire d'élimination des guérillas par n'importe quel moyen ${ }^{8}$. Les forces armées, dès les années 1960, ont, non seulement toléré, mais ont aussi travaillé avec des groupes armés illégaux appelés autodéfenses afin d'éradiquer les guérillas. Elles ont depuis cette époque instauré une politique systématique de guerre avec un budget militaire en augmentation. La raison invoquée est ici de reprendre le contrôle des territoires perdus et de représenter la conscience populaire mécontente. Les autodéfenses se sont proposées de refonder le pays et de consolider un modèle social et politique oligarchique et mafieux avec le soutien du paramilitarisme, qui a connu un processus d'expansion nationale grâce aux contributions financières directes de la part des éleveurs de bétail, des industriels et des entrepreneurs internationaux ${ }^{9}$.

Ces alliances sont à la base du développement d'un véritable "bras armé" de l'État et de plusieurs formes de lutte qui ont entraîné une profonde dégradation du conflit armé, caractérisé par des persécutions systématiques et une politique d'assassinats contre des civils appartenant à des mouvements sociaux et syndicaux progressistes, comme l'Uñion patriotica (Union patriotique - UP) qui a joué un rôle politique majeur dans la première décennie des années 1980. Le fait que quelques insurgés réinsérés dans la vie civile militaient au sein de l'UP a été mis en avant par les forces militaires et a servi d'excuse pour étendre la suspicion à tous les membres de cette organisation populaire. Ce parti a réussi à se développer politiquement

4. R. Arango, «Cinco años de justicia y paz... »: «L'absence d'un système adéquat ainsi que des institutions insuffisantes pour surveiller l'accomplissement des conditions légales par les condamnés pour profiter d'avantages promet des décennies de procédures pénales de justice et de paix avec des résultats incertains et générant très probablement un degré élevé d’impunité. La faiblesse historique du système de justice explique son incapacité à gérer les 4000 cas des mis en examen, et encore davantage son incapacité à traiter ceux de plus de 27000 paramilitaires qui restent dans les limbes afin de les rendre responsables des délits commis, ou pour assurer une réparation intégrale des victimes. Tout se dirige vers un échec coûteux, dissimulé sous la façade d'indemnisations en millions par l'État, sous les fables du gouvernement évoquant un processus inédit et sous le camouflage des véritables responsabilités des militaires, des propriétaires fonciers, des industriels et des politiciens».

5. A. Peña, « ¿ Existe una política para combatir las bandas emergentes en Colombia ?», Semana, 11 février 2010, disponible à l’adresse: http://www. semana.com/noticias-seguridad/existe-politica-para-combatir-bandas-emergentes-colombia/134839.aspx; «Les bandes criminelles ne sont pas le seul problème pour le gouvernement; il existe désormais le problème de la médiation dans certains secteurs, afin que les bandes limitent le volume de la violence générée dans les villes. Ces groupes, qui ont commencé à apparaître après la démobilisation des paramilitaires, estiment rassembler environ 4000 hommes répartis dans 248 municipalités à travers le pays [...]». Récemment, l'organisation internationale de protection des droits de l'homme Human Rights Watch a évoqué la nouvelle forme d'organisation qu'ont pris les paramilitaires, dans un rapport intitulé «Herederos de los paramilitares» (3 février 2010, disponible à l'adresse: http://www.hrw.org/en/node/88063/section/4); «le gouvernement colombien et certains analystes qualifient les groupes successeurs de "bandes criminelles émergentes au service du narcotrafic (ou Bacrim)", et insistent sur le fait que ces groupes nouveaux sont un phénomène nouveau et totalement différent des paramilitaires. D’autres experts considèrent que ces groupes sont une continuité des AUC [Autodéfenses unies de Colombie], ou une nouvelle génération de paramilitaires. Bien qu'il existe des différences entre les AUC et les groupes successeurs, ces derniers constituent en fait la suite de certains des "blocs" ou des groupes paramilitaires. Tel que la police l'a rapporté, presque tous les chefs des groupes successeurs sont des chefs des AUC, classés à des postes médians d'un point de vue de la hiérarchie des groupes paramilitaires, qui n’ont jamais été démobilisés, ou qui continuent à participer à des activités criminelles bien qu'ils affirment être démobilisés. Les groupes sont actifs dans plusieurs régions, identiques à celles dans lesquelles les AUC étaient présentes, etfonctionnent d'une manière similaire à celles-ci: contrôle des territoires au moyen de menaces et d'extorsions, participation au trafic de drogues et à d'autres activités criminelles et violations généralisées contre les civils ».

6. Voir le rapport d'experts sur l'emploi, la pauvreté et l'inégalité, Bogota, 24 août 2009, disponible à l'adresse: http://web.presidencia.gov.co/ sp/2009/agosto/24/08242009.html.

7. Voir M. V. Uribe, Salvo el poder todo es ilusión. Mitos de origen: tigres tamiles de Sri Lanka, fuerzas armadas revolucionarias de Colombia, Irish Republican Army, Bogota, Institut Pensar, Pontificia Universidad Javeriana, 2007, p. 61-87.

8. Voir M. G. Magil, Crónica oculta del conflicto. Estrategias de impunidad en la narcoparapolítica. El acuerdo humanitario, los desaparecidos... y el fin de la guerra, Bogota, Ediciones desde abajo, 2008, p. 61-62.

9. Voir «El verdadero jefe de las autodefensas le da la cara al país por primera vez», entretien avec Vicente Castaño, Semana, 5 juin 2005 , p. 30. 
dans une grande partie du pays et a eu un impact social et politique profond sur les institutions, jusqu'à représenter une véritable option de pouvoir au début des années 1980.

La montée de l'organisation populaire est devenue une menace pour la consolidation de l'alliance Étatparamilitarisme-narcotrafic. Par conséquent, des politiques et des stratégies d'élimination des dirigeants sociaux ont été mises en place, en principe appliquées de façon sélective. Le développement historique et l'expansion de la base militaire ont toutefois conduit les organisations sociales et communautaires à devenir un instrument de contrôle social, d'accusation, de surveillance et de mort. Sous l'égide de divers programmes institutionnels de soutien social et économique promus par la présidence de la République en vertu de la politique dite de «sécurité démocratique», des avantages et subventions ont commencé à être donnés à partir de 2002 aux familles et informateurs qui étaient au chômage.

Cette nouvelle forme de reconstruction sociale a conduit à une croissance sans précédent de cette nouvelle matrice de contrôle social, qui a abouti à limiter l'autonomie politique et sociale des citoyens et de leurs familles alors convertis en réceptacle de "faveurs et cadeaux" donnés par l'État en tant que substitut des droits sociaux et de la sécurité sociale, qui tendaient à disparaître sous le régime de la soi-disant flexibilité du travail.

Dans cet ordre d'idées, il convient de noter que les autodéfenses ont été créés par l'État dans un cadre juridique spécial figurant dans le décret 3398 de 1965, adopté comme législation permanente avec la loi 48 de 1968. Cette norme contre l'insurrection a permis aux civils de s'armer, y compris avec des armes à usage exclusif des forces armées, comme l'a rappelé la Cour interaméricaine des droits de l'homme dans son troisième rapport sur la situation des droits de l'homme:

[...] l'État a joué un rôle important dans le développement des groupes appelés paramilitaires d'autodéfense, en leur permettant d'agir avec une protection légale et une légitimité dans les années soixante et quatre-vingt, et il est responsable d'une manière générale [...]. Résultat de la politique de contre-insurrection, les paramilitaires ont établi des liens avec l'armée colombienne, liens qui se sont renforcés durant plus de deux décennies. Finalement, le 25 mai 1989 la Cour de cassation a déclaré inconstitutionnelle la loi 48 de 1968, supprimant le cadre juridique légal qui liait les groupes paramilitaires ou les autodéfenses avec la défense nationale [...]. Malgré cela, l'État a fait peu pour démanteler la structure qu'il avait créée et favorisée, en particulier lorsque ces groupes commandaient des activités anti-insurrectionnelles [...]. Profitant du pouvoir des armes et de la couverture qui avait été donnée par l'État à ces activités illégales, ils se sont tournés vers des activités illicites aussi productives que le trafic de drogue, les enlèvements, le déplacement forcé, l'extorsion, ce qui leur a accordé un énorme pouvoir économique et leur a permis le contrôle militaire de zones spécifiques du territoire, le déploiement d'actions visant la population civile comme victime du conflit, en les soumettant à des pratiques de terreur qui se traduisent par des violations graves, massives, systématiques et répétées des droits de l'homme et des règles du Droit International Humanitaire ${ }^{10}$.

La coopération internationale a contribué à de tels projets, avec des financements, entre autres, par les États-Unis à travers le plan Colombie, le plan Patriote et d'autres accords de coopération souscrits avec l'Union européenne, ressources destinées à éradiquer les guérillas des Forces armées révolutionnaires de Colombie (FARC $)^{11}$ et à reprendre le contrôle de la société au travers de la distribution de sommes d'argent sous forme de dons fournis par des projets comme «Familles Garde Forêts ", qui sont également devenus des stratégies politiques électorales.

Cette tactique, qui s'est imposée dans tous les espaces de la vie quotidienne et est devenue un véritable mode de vie, a été le ferment d'une culture de mort et de suspicion à l'encontre des personnes et des groupes qui s'opposent et dénoncent ce type de politiques. Au cours des quatre dernières décennies, des milliers de Colombiens ont été victimes de disparitions, déplacements, massacres, violations des droits de l'homme et assassinats. À propos de l'alliance paramilitarisme - forces de l'État, la Commission interaméricaine des droits de l'homme a indiqué que la création de groupes paramilitaires avait été motivée par l'État comme un outil de lutte anti-insurrectionnelle sous la protection de normes légales et que,

[...] dans les cas où des paramilitaires et des membres de l'armée conduisent des opérations conjointes ou lorsque les paramilitaires opèrent avec l'acquiescement [ou] la collaboration de la force publique, on se doit de considérer que les membres des groupes paramilitaires agissent comme agents de l'État [...].

Elle ajoute:

[...] sont imputables à l'État à la fois les violations de la Convention américaine résultant des actes et omissions de ses propres agents, comme celles commises par des membres d'un groupe d'autodéfense qui opérait dans la région avec leur soutien et, à cette fin, doivent être considérés comme des agents de l'État ${ }^{12}$.

La mise en œuvre de cette politique d'extermination et de consolidation du nouveau modèle de la patrie a été rendue possible par de fortes collusions entre les politiques et les paramilitaires. Cela a été mis en évidence par le

10. S. Mora Insuasty, La ley de Justicia y Paz en el marco de la Convención americana de derechos humanos, Bogota, Ediciones Nueva Jurídica, 2008, p. 18.

11. Voir H. Faivre d'Arcier-Flores, «Del Plan Colombia al Plan Patriota: impacto del conflicto armado colombiano en el Ecuador, análisis de una estrategia de seguridad subregional ", Revista complutense de historia de América, vol. 31, 2005, p. 215-230 (p. 222), disponible à l'adresse: revistas. ucm.es/index.php/RCHA/article/download/RCHA0505110215A/28579.

12. Cour interaméricaine des droits de l'homme, affaire du massacre de La Rochela c. Colombie, arrêt du 11 mai 2007, $\$ 71$, disponible à l'adresse : http://www.corteidh.or.cr/docs/casos/articulos/seriec_163_esp.pdf. 
procès de plus de cent députés jugés en raison de leurs liens avec les paramilitaires, ainsi qu'en raison des liens existant entre le pouvoir exécutif et les actions illégales du $\mathrm{DAS}^{13}$, organisme qui relève directement de la présidence de la République, et dont les actes représentent «l'assujettissement d'une institution aux intérêts capricieux des chefs paramilitaires et des trafiquants de drogue ${ }^{14}$. Un exemple pertinent de cette politique de l'État est le rôle de Jorge Noguera, directeur du DAS nommé par le président Álvaro Uribe lors de son premier mandat pour diriger les services de renseignement de l'État. Ce personnage, dans le cadre d'une procédure pénale, a été interdit d'exercer des fonctions publiques pendant 18 ans par le Bureau du Procureur général en 2007, étant accusé de collaborer à la création et au soutien de groupes armés hors la loi au cours de son administration ${ }^{15}$.

\section{La stratégie de constitution et de consolidation du modèle politique de guerre}

Entre 1983 et 1987, la stratégie de guerre de l'État a fait plus de 25000 morts au sein de l'UP et elle se poursuit encore aujourd'hui avec des assassinats ciblés et des déplacements massifs vers des zones qui possèdent de grandes ressources naturelles, des élevages, des monocultures, des plantes et permettent ainsi les actions du narcotrafic et son implication dans la politique. Pour développer cette politique, comme l'a affirmé Vicente Castaño, il était nécessaire que tous les commandants apportent la richesse dans l'ensemble des régions du pays, afin de développer de nouveaux modèles d'entreprises au niveau national. Cette expansion d'entreprises, promue de manière stratégique par les AUC, a été mise en œuvre à travers la dépossession agraire dans l'Urabá chocoano, avec la participation de Freddy Rendón Henao et d'autres chefs des autodéfenses. Ils ont ainsi étendu dans la municipalité de Belén de Bajirá le rentable projet de culture d'huile de palme pour produire le biodiesel - le carburant $\mathrm{du} \mathrm{XXI}^{\mathrm{e}}$ siècle -, fortement soutenu par le gouvernement d'Álvaro Uribe dans tout le pays ${ }^{16}$.

Le déploiement de cette action étatique, combiné à l'absence d'un statut de l'opposition politique et démocratique qui aiderait à garantir les droits politiques, a d'abord conduit à transformer les partis d'opposition en objectifs militaires, puis a mené à leur disparition, en même temps que se consolidait un nouveau modèle d'État mafieux, avec le pacte de Ralito de 2001, ayant pour objectif de «refonder la Patrie», intention matérialisée et légitimée par l'élection d'Álvaro Uribe Vélez à la présidence de la Colombie pour la période 2002-2006. Son mandat a finalement été prolongé jusqu'en 2010 et poursuivi par son successeur et héritier, ancien ministre de la Défense, Juan Manuel Santos, qui a repris sa politique de sécurité, avec quelques évolutions propres à un modèle économique axé sur la mobilité des capitaux transnationaux ${ }^{17}$.

Le processus de légitimation institutionnelle des principes du paramilitarisme comme forme politique de l'État a commencé avec la campagne présidentielle d'Álvaro Uribe Vélez (années 2001 et 2002), lorsqu'il a demandé le soutien des groupes paramilitaires des AUC pour arriver au gouvernement et mettre en œuvre la politique de sécurité démocratique ${ }^{18}$. Il leur avait alors promis une négociation qui leur permettrait de rejoindre la vie civile et politique à partir du moment où auraient été mises en place les conditions nécessaires pour le renforcement de l'État et de ses institutions.

Le pacte politique prétendait régler la dette avec le paramilitarisme, fermant ainsi temporairement les portes au processus de négociation avant l'entrée en vigueur de la compétence de la Cour pénale internationale (CPI) pour pouvoir connaître les crimes de guerre (en novembre 2009). Lorsque le président Andrés Pastrana avait signé le Statut de Rome en août 2002, il avait fait appliquer l'article 124 dudit traité international en accord avec le président nouvellement élu Álvaro Uribe et, par conséquent, exclu la compétence de la CPI pour connaître ce type de délits en Colombie pendant sept ans ${ }^{19}$.

13. Departamento administrativo de seguridad: service de renseignement colombien fondé en 1953 et dissous le 31 octobre 2011 par le décret 4057 de 2011, dont les fonctions ont été déléguées à d'autres services de l'État.

14. Observatorio de derechos humanos y derecho humanitario [Observatoire des droits de l'homme et du droit humanitaire], La pesadilla del DAS. Recuento del "huracán político», desde Semana Santa hasta la víspera de Navidad, para comenzar a discutir la depuración y reforma profundas que requieren los organismos de inteligencia colombianos, Bogota, Coordinación Colombia-Europa-Estados Unidos (Documentos temáticos, $\left.\mathrm{n}^{\circ} 3\right), 2006$, p. 8 .

15. Le Bureau du Procureur général, qui a adopté la mesure, a été lui aussi interdit d'exercer des fonctions pendant 18 ans; voir «Destituido ex director de DAS Jorge Noguera", El espectador, 16 novembre 2007, disponible à l'adresse: www.elespectador.com/noticias/judicial/articulodestituido-ex-director-de-das-jorge-noguera.

16. Voir N. Quevedo H., J. D. Laverde P., «En la ruta de los Castaño. El lío de los títulos colectivos de Jiguamiandó y Curvaradó», El espectador, 19 février 2008, disponible à l'adresse : http://www.elespectador.com/impreso/cuadernilloa/investigacion/articuloimpreso-ruta-de-los-castano?page=o,2; «Les mains du narcotrafic et du paramilitarisme sont visibles dans le développement de l'industrie de la culture de palme dans la zone de l’Urabá chocoano. La région connaît actuellement des moments de tension alors que sont menées des enquêtes par le Procureur».

17. Le chef paramilitaire Mancuso avait affirmé il y a quelques années qu'en cas d'élection d'Álvaro Uribe Vélez cela serait plus facile car son discours était semblable au leur et il avait recommandé aux électeurs de voter pour lui. Voir «Mancuso se destapa», El tiempo, 16 janvier 2007, disponible à l'adresse: http://www.eltiempo.com/archivo/documento/MAM-2359666.

18. Voir presidencia de la República, Oficina del alto comisionado para la paz [présidence de la République, Bureau du haut-commissaire pour la paix], Proceso de paz con las autodefensas, Bogota, décembre 2006, Présentation générale, p. 5.

19. "Con la entrada de la Corte Penal Internacional se cerraron puertas para diálogo con la guerrilla", Cambio, août 20o6, disponible à l'adresse: http://www.cambio.com.co/paiscambio/840/ARTICULO-WEB-NOTA_INTERIOR_CAMBIO-578429o.html. 
Le président Uribe, tout au début de son premier mandat, avec le projet de référendum soumis au Congrès et approuvé en tant que loi 796 de 2003, en est même arrivé à faire adopter dans le paragraphe $n^{\circ} 6$, qui s'ajoutait à l'article 176 de la Constitution, un dispositif permettant de nommer directement - et de façon permanente - dans les assemblées représentatives nationales et locales, les chefs paramilitaires qui acceptaient le processus ${ }^{20}$.

À partir du $1^{\text {er }}$ décembre 2002, les groupes paramilitaires AUC, BCB (Bloc central Bolivar) et Alliance orientale ont déclaré unilatéralement la cessation des hostilités dans un accord à portée nationale; par la suite, le 15 juillet 2003, la phase de négociation a commencé, avec l'accord de Santa Fe de Ralito pour contribuer à la paix en Colombie, dans lequel les groupes paramilitaires s'engageaient à démobiliser tous leurs membres avant le 31 décembre 2005 . Le désarmement a duré jusqu'au 15 août 2006 et tout au long de ce processus 31671 hommes ont été démobilisés. La deuxième phase consistait en l'engagement des chefs paramilitaires à avouer la vérité. Suite à la décision des paramilitaires de déposer les armes et de se conformer à la loi de Justice et de Paix, plusieurs enquêtes ont été diligentées visant à évaluer l'efficacité du processus et à déterminer le bon fonctionnement de la justice dans le cadre de sa lutte contre l'impunité.

L'internationaliste Kai Ambos, dans son étude sur la faculté d'intervention complémentaire de la CPI à la lumière du dénommé processus de Justice et de Paix en Colombie $^{21}$, fondée sur la loi 975 de 2005 dite de Justice et de Paix et écrite sous l'influence de l'article $17 \mathrm{du}$ Statut de Rome, estime que les processus se sont déroulés de manière lente mais conforme à la loi, de sorte que l'on ne peut considérer qu'il existe une impunité absolue, même si une seule condamnation avait été prononcée à la date du rapport. Des efforts ont cependant été déployés pour faire avancer le processus. Le point de vue de l'internationaliste est conforme aux données que le Ministère public a transmis à la Comisión nacional de reparación y reconciliación (Commission nationale de réparation et réconciliation - CNRR), qui constate qu'à ce jour un seul arrêt de condamnation a été prononcé, tandis que le nombre de victimes enregistrées dépasse les 300 ooo, en plus d'autres situations mettant en doute le sérieux du processus de Justice et de Paix.

En outre, le Bureau du Procureur général a certifié qu'au 30 décembre 2010 aucun politicien n'avait été mis en examen en vertu de la loi de Justice et de Paix, raison pour laquelle l'Unité nationale des procureurs pour la Justice et la Paix a renvoyé à la justice ordinaire 429 dossiers de politiciens, afin que les juridictions ordinaires ou la Cour suprême de justice (chambre criminelle de la Cour de cassation) puissent faire avancer les investigations correspondantes ${ }^{22}$.

La manière dont a été négocié le processus de Justice et de Paix, les irrégularités dans le processus de démobilisation et de réinsertion et l'adoption d'une loi de Justice et de Paix avec des sanctions limitées qui laissent presque dans l'impunité la grande majorité des crimes, stigmatisent l'ordre juridique colombien, maintiennent le pays dans un état d'anxiété face à la violation continue des droits de l'homme et génèrent un état de crise permanente. Cette situation mérite une analyse approfondie du travail des institutions qui ont élaboré un ensemble de règles dérogatoires destinées à légitimer la réinsertion. Le déroulement des processus démontre des anomalies dans l'application de la loi, au regard des standards internationaux des droits de l'homme.

\section{Les progrès du processus de Justice et de Paix face au paramilitarisme}

La loi 975 de 2005 a été présentée comme permettant, de manière adéquate, d'enquêter, d'identifier, de poursuivre et de punir les paramilitaires responsables de l'actuelle crise humanitaire, et de combattre l'impunité, afin de rétablir les droits de ceux qui ont souffert de la violation systématique de leurs droits fondamentaux. Ces multiples actions se sont traduites par des réductions ou substitutions de peine pour ceux qui ont été mis en cause, l'objectif étant d'atteindre un équilibre apparent entre la vérité et la justice. Les mesures accordaient en outre aux délinquants la possibilité de profiter d'avantages spéciaux dans le cadre de comportements criminels d'une extrême gravité.

La règle n'a pas inclus de mécanismes juridiques permettant d'établir une pleine clarification des faits criminels et de connaître la vérité sur ce qui s'est réellement passé. Sans le respect de ces conditions il n'est pas possible de parler d'une justice effective et encore moins d'assurer aux victimes une réparation intégrale permettant la jouissance de leurs droits. Il n'a pas plus été exigé de confession qui permettrait, au moins, d'approcher de la

20. «Ley 796 de 2003", Diario oficial, no 45.076, disponible à l’adresse: http://www.elabedul.net/Documentos/Leyes/2003/Ley_796.pdf; «Afin de faciliter le retour à la vie civile des groupes armés hors la loi qui se sont trouvés liés au processus de paix mis en place par le gouvernement, des districts spéciaux de paix pour les élections des corporations publiques réalisées avant le 7 août 2006 pourront être établis - pour une seule fois -, ou un nombre proportionnel de membres du Congrès, de députés et de membres des groupes mentionnés dans le processus de paix et démobilisés pourra être nommé directement - pour une seule fois. Le nombre sera fixé par le gouvernement national, en prenant en compte les circonstances et le déroulement du processus. Les noms des membres du Congrès, des députés et des conseillers visés au présent article seront déterminés entre le gouvernement et les groupes armés, et leur désignation sera assurée par le président de la République».

21. K. Ambos, Procedimiento de la ley de Justicia y Paz (ley 975 de 2005) y derecho penal internacional: estudio sobre la facultad de intervención complementaria de la Corte penal internacional a la luz del denominado proceso de "Justicia y Paz" en Colombia, Göttingen, Université GeorgAugust, 2010, p. 6, disponible à l'adresse: http://www.department-ambos.uni-goettingen.de/index.php/component/option,com_docman/ Itemid,71/gid,171/task,cat_view.

22. Unidad nacional de fiscalías para la Justicia y la Paz [Unité nationale des procureurs pour la Justice et la Paix], document UNJP nº o18666, Bogota, 30 décembre 2010 (en réponse au droit de pétition de cette équipe de recherche). 
vérité historique, avec la mise en place de moyens adaptés pour vérifier ou infirmer la version de ceux qui sont mis en cause. L'obligation de coopérer efficacement avec la justice en fournissant toute information ou preuve de la participation de démobilisés ou de leurs groupes, qui permettrait d'envisager un démantèlement réel et effectif des structures illégales, est un autre mirage. Comment alors réfléchir à la lutte contre l'impunité et consolider un véritable processus de paix?

La loi ne fournit pas suffisamment de mécanismes pour obliger les criminels à rendre les biens arrachés à leurs victimes de manière violente dans de nombreux endroits du territoire national, pas plus qu'elle n'envisage comment doivent être réparés les dommages causés aux personnes déplacées tant individuellement que collectivement et qui se retrouvent aujourd'hui dans des conditions de vulnérabilité. Cette norme prévoit d'ailleurs une application très large et manifestement disproportionnée des peines privatives de liberté: pour la commission de crimes graves et préjudiciables à la dignité humaine, elle établit une échelle s'étalant de 5 à 8 ans de privation de liberté, avec des possibilités de liberté conditionnelle, sans mécanismes pour contraindre l'auteur à expliquer ses faits criminels. Ces «oublis» se sont traduits par une totale impunité, le non-respect des principes constitutionnels et la non-application des normes internationales relatives aux droits de l'homme et au droit international humanitaire.

D’autre part, la loi de Justice et de Paix a été appliquée de manière marginale à tous ceux qui avaient été démobilisés, en considérant que leur situation juridique n'avait pas été prise en compte par la loi 782 de 2002 (loi qui s'applique aux auteurs de délits graciables ou amnistiables, comme la rébellion) et son décret d'application 128 de 2003. La loi 975 reprend toutefois expressément l'énoncé de la loi 782 qui évoque des «dispositions pour faciliter le dialogue et la signature d'accords avec les groupes armés hors la loi pour la démobilisation, la réconciliation entre les Colombiens et la coexistence pacifique ». Dans son introduction, la loi 975 détermine comme objectif de «contribuer efficacement à la réalisation de la paix nationale». Dans l'article $1^{\mathrm{er}}$, qui annonce l'objet de la loi, il est indiqué que:

Cette loi est destinée à faciliter le processus de paix et de réintégration individuelle ou collective à la vie civile des membres de groupes armés hors la loi, garantissant les droits des victimes à la vérité, la justice et la réparation ${ }^{23}$.

La condition pour pouvoir bénéficier d'une peine alternative était de collaborer efficacement au processus de paix national, et faire ainsi de la vérité l'élément central pour la réparation des victimes et la prévention de nouveaux délits. La conséquence logique du processus de paix était la mise en place de conditions empêchant la répétition des exactions, la guérison des blessures produites dans le passé et la prévention des comportements criminels dans l'avenir, ce qui correspond à la théorie pénale contemporaine et aux standards nationaux et internationaux qui réglementent ces questions.

De ce qui a été annoncé rien ne s'est passé:

[...] le gouvernement d'Uribe a perdu la direction de la réincorporation de ces forces à la vie civile, car il n'a pas agi avec honnêteté et transparence dans l'application de la loi, et il n'a pas reconnu depuis le début qu'un vaste réseau d'agents de l'État et des dirigeants politiques des partis de la coalition du gouvernement faisaient partie des paramilitaires; il n'a pas accepté non plus de reconnaître que les paramilitaires étaient très impliqués dans le trafic de drogue. Il a voulu tromper l'opinion publique, en présentant les paramilitaires comme relevant d'un projet fondamentalement révolutionnaire ${ }^{24}$.

Dans cet ordre d'idées, lorsque l'activité de l'exécutif colombien a été mise en doute dans le cadre de pressions internationales sur les progrès et les réalisations de la loi de Justice et de Paix, Uribe a décidé d'extrader les chefs paramilitaires aux États-Unis, en essayant de protéger les dirigeants politiques impliqués dans la grave crise humanitaire, bloquant ainsi à plusieurs moments le travail du Procureur et de la Cour suprême de justice. L'exécutif a cherché à éviter une mise en cause de la responsabilité de l'État face à la terrible tragédie qui a touché une grande partie de la population dans différentes régions. L'exécutif n'a pas initié, non plus, les réformes institutionnelles nécessaires, pas plus qu'il n'a présenté d'initiatives au Congrès en vue de mettre en ouvre la réparation et la reconstruction du pays.

La décision d'Uribe Vélez de mai 2008 d'extrader vers les États-Unis les chefs paramilitaires les plus représentatifs pour affronter les accusations de trafic de drogue, blanchiment d'argent et terrorisme, alors qu'ils relevaient de l'application de la loi de Justice et de Paix, a été reçue avec surprise. Il était en effet prévu qu'au cours de l'enquête des révélations auraient pu être faites au sujet des liens existants entre les structures paramilitaires et les groupes économiques et sociaux les plus puissants et les plus influents du pays, des entreprises nationales, des hauts fonctionnaires au service de l'État à différents niveaux, les membres de la force publique, les élus du Congrès de la République et d'autres institutions, tous appartenant à des secteurs très proches de l'administration de l'État. L'explication est simple: en les soustrayant à la juridiction nationale et en privilégiant une juridiction étrangère, il était possible de favoriser une impunité totale et donc de cacher la vérité ou toute possibilité de la connaître.

La chambre criminelle de la Cour de cassation, dans son arrêt du 21 septembre 2009, dans le cadre de la procédure de Justice et de Paix contre Gian Carlo Gutiérrez Suárez, démobilisé du Bloc Calima des AUC, a reconnu que les individus ayant parrainé, orienté, diffusé et aidé 
à consolider et renforcer, mais aussi servi les groupes paramilitaires, faisaient partie intégrante des structures des AUC. Ils doivent donc répondre de l'actuelle crise humanitaire, les divers secteurs de la société colombienne, comme les dirigeants politiques, les agents de l'État à travers le pays et les leaders locaux, ayant tissé un véritable réseau d'actions criminelles.

Selon cet arrêt, le nombre de personnes impliquées dans les agissements du paramilitarisme et qui ont contribué d'une façon ou d'une autre à mettre en place d'anciennes et sombres alliances rassemblant des acteurs publics comme privés serait très élevé. Ces alliances ont indéniablement réussi à se consolider et à se radicaliser au cours de l'ère de la sécurité démocratique. Camilo Castellanos, suivant la thèse de Luis Jorge Garay, a estimé que nous assistions à la « reconfiguration par l'action de l'État d'une mafia narco-politique et paramilitaire en gestation depuis au moins deux décennies». Cette même mafia constitue selon lui un bloc de pouvoir, ce qui signifie que «dans la première décennie du XXI siècle, la Colombie a régressé ${ }^{25}$. De même, Alejandro Mantilla affirme que l'actuel régime en Colombie se distingue par son caractère prémoderne parce que «[...] la loyauté n'a pas besoin de tout ce qui est public [...], seule compte la loyauté avec le chef de l'État ${ }^{26}$.

L'administration d'Uribe, avec sa politique de sécurité démocratique, s'est distinguée par son utilisation détournée des réformes législatives et judiciaires afin d'aider de nombreux hommes politiques qui appartenaient au gouvernement et étaient poursuivis judiciairement. De plus, durant ce mandat d'Uribe, diverses formes de violation des droits de l'homme et de crimes contre l'humanité se sont développées, telles que les situations de «falsos positivos» («faux positifs») ${ }^{27}$, existant dans différentes zones du territoire.

Les chiffres traduisant les progrès en matière de justice, vérité et réparation pendant les années d'application du processus de Justice et de Paix, le nombre d'individus réinsérés, les résultats des procédures judiciaires et l'application des droits des victimes, sont présentés dans les tableaux statistiques suivants et démontrent de manière incontestable l'inefficacité du processus de paix.

25. Plataforma colombiana de derechos humanos, democracia y desarrollo [Plate-forme colombienne pour les droits de l'homme, la démocratie et le développement], ¿Continuidad o desembrujo? La seguridad democrática insiste y la esperanza resiste. Siete años de gobierno de Álvaro Uribe Vélez, Bogota, Plataforma colombiana de derechos humanos, democracia y desarrollo, 2009, p. 6.

26. Ibid.

27. L'expression «faux positifs» renvoie aux assassinats de civils innocents effectués par des membres de l'armée nationale colombienne, en les faisant passer pour des guérilleros morts au combat dans le cadre du conflit armé. Selon le droit international, ces cas sont des exécutions extrajudiciaires, et selon le droit pénal colombien, il s'agit d'homicides sur personne protégée. 


\section{Annexe:}

\section{Tableaux et analyses de l'application de la loi de Justice et de Paix cinq ans après sa mise en place ${ }^{28}$}

Jusqu'en 2010, la loi de Justice et de Paix n'a guère donné de résultats comme l'information transmise par l'Unité nationale des procureurs pour la Justice et la Paix permet de le constater.

Dans le premier tableau est enregistré l'état général des procédures engagées. Il permet ainsi de constater que plus de $60 \%$ des individus ont été protégés par le principe d'opportunité selon la loi 1312 de $2009^{29}$. D'autre part, l'extradition de certains chefs a sérieusement affecté le droit des victimes à connaître la vérité. Ainsi, sur les 3000 cadavres exhumés, seuls 908 corps ont été identifiés.

Dans le deuxième tableau figurent les informations traitées par le Bureau du Procureur général au 30 novembre 2010, sur l'application et la situation des individus qui ont été mis en examen sur la base de la loi 975 de 2010.

Le troisième tableau présente le nombre d'individus mis en examen, la quantité d'incidents de réparation ${ }^{30}$ présentés et les arrêts de condamnations.

Enfin, les tableaux 4 et 5 relatent les types de délits qui sont soumis dans les procédures de la loi de Justice et de Paix, plus particulièrement les délits démontrés par la reconnaissance d'individus impliqués.

Tableau 1: État de la situation des procédures de Justice et de Paix en décembre 2010

\begin{tabular}{|l|c|}
\hline \multicolumn{1}{|c|}{$\begin{array}{c}\text { Procédures menées dans le cadre } \\
\text { de la loi de Justice et de Paix }\end{array}$} & Nombre de personnes \\
\hline Anciens combattants favorisés par la loi de Justice et de Paix & 2872 \\
\hline Combattants irréguliers démobilisés collectivement & 2161 \\
\hline Chefs de groupes paramilitaires jugés & 670 \\
\hline Détenus au moment de la démobilisation & \\
\hline Individus qui se sont vus notifier leur mise en examen & 1790 \\
\hline $\begin{array}{l}\text { Individus ayant fourni des déclarations libres devant les } \\
\text { procureurs adjoints de Justice et de Paix }\end{array}$ & 641 \\
\hline Déclarations libres réalisées de manière informelle & \\
\hline Déclarations libres en cours & \\
\hline
\end{tabular}

28. Voir «Ley de justicia y paz: cinco años de impunidad», Agencia de prensa IPC, 26 juillet 2010, disponible à l'adresse: http://www.ipc.org.co/agenciadeprensa/index.php?option=com_content\&view=article\&id=417:leyde-justicia-y-paz-cinco-anos-de-impunidad\&catid=78:general\&Itemid=176.

29. Texte qui a modifié la loi 906 de 2004 (Code de procédure pénal colombien) par rapport au principe d'opportunité.

30. La loi pénale colombienne accorde aux victimes, entre autres, le droit à une réparation rapide du préjudice subi en raison de la conduite criminelle de l'auteur ou d'un tiers responsable. L'étape mise en place par le nouveau système pénal accusatoire, pour faire valoir ce droit, est «l'incident de réparation», qui est la phase suivant l'arrêt de condamnation, ce qui signifie qu'il est nécessaire que le juge déclare préalablement par un arrêt la responsabilité pénale de l'accusé. À ce stade de la procédure pénale, la victime doit prouver les dommages subis, pour pouvoir, à travers un règlement à l'amiable ou une décision du juge, obtenir une réparation en totalité ou en partie des dégâts subis. 


\begin{tabular}{|c|c|}
\hline $\begin{array}{l}\text { Arrêt de condamnation au groupe Héroes de los Montes de } \\
\text { María dans le cadre du massacre de Mampuján }{ }^{31}\end{array}$ & 1 \\
\hline $\begin{array}{l}\text { Individus protégés par le principe d'opportunité selon la } \\
\text { loi } 1312 \text { de } 2009\end{array}$ & $\begin{array}{c}19000 \\
\text { (plus de 60\%) }\end{array}$ \\
\hline $\begin{array}{l}\text { Individus pouvant être amnistiés par le Procureur en applica- } \\
\text { tion du principe d'opportunité, par suspension, interruption } \\
\text { ou renonciation à l'enquête }\end{array}$ & 31700 \\
\hline Individus réinsérés toujours en vie & 3000 \\
\hline Individus réinsérés assassinés & 820 \\
\hline Individus récidivistes dans des comportements criminels & 3000 \\
\hline $\begin{array}{l}\text { Personnes disparues au cours des trois dernières années selon } \\
\text { le rapport CNRR basé sur le rapport de médecine légale }\end{array}$ & 38025 \\
\hline $\begin{array}{l}\text { Total des personnes disparues figurant dans le Registre } \\
\text { national des disparus en novembre } 2010\end{array}$ & 51300 \\
\hline $\begin{array}{l}\text { Disparitions forcées sur le total cumulé des personnes dis- } \\
\text { parues figurant dans le Registre national des disparus en } \\
\text { novembre } 2010\end{array}$ & 12600 \\
\hline $\begin{array}{l}\text { Cas d'éventuelles exécutions extrajudiciaires en Colombie } \\
\text { selon le haut-commissaire }\end{array}$ & 2500 \\
\hline Victimes enregistrées & 288661 \\
\hline Chefs (capos) extradés & 13 \\
\hline $\begin{array}{l}\text { Envois des dossiers à la justice ordinaire, données fondées sur } \\
\text { les versions libres réalisées par des parapoliticiens (gouver- } \\
\text { neurs régionaux, députés, fonctionnaires publics) }\end{array}$ & 350 \\
\hline $\begin{array}{l}\text { Exhumations de cadavres de fosses communes, données } \\
\text { fondées sur la reconnaissance des actions criminelles }\end{array}$ & 3000 \\
\hline $\begin{array}{l}\text { Ouvertures de procédures contre des politiciens pour para- } \\
\text { politique }\end{array}$ & 350 \\
\hline Procès engagés contre des membres des forces armées & 220 \\
\hline Procès engagés contre des civils & 5000 \\
\hline
\end{tabular}

Aux données partielles et approximatives mentionnées ci-dessus s'ajoutent les résultats tirés du rapport demandé à l'équipe de recherche «Libertad y Garantismo », et transmis à l'Unité nationale des procureurs pour la Justice et la Paix, en date du 30 novembre 2010, et qui complètent et consolident l'information identifiée ${ }^{32}$.

31. Le massacre de Mampuján fait référence aux faits qui se sont déroulés dans la nuit du 10 au 11 mars 200o. Les paramilitaires du bloc Héroes de los Montes de Maria ont envahi le village les Brisas, qui jouxte le village de Mampuján. Ils ont assassiné douze paysans en les signalant comme des collaborateurs de la guérilla. Après le massacre, ils ont menacé l'ensemble de la population, conduisant au déplacement forcé de 180 familles.

32. Unidad nacional de fiscalías para la Justicia y la Paz [Unité nationale des procureurs pour la Justice et la Paix], document UNJP no 018 665, Bogota, 30 décembre 2010, adressé à Lina Mercedes Guzman en réponse à l'information demandée le 30 novembre 2010 , dans le processus de vérification et de consolidation. Ce document relève que cette Unité nationale a reçu du gouvernement $4484 \mathrm{mis}$ en examen dans le cadre de l'application de la loi 975 de 2005 
Tableau 2: Informations traitées par le Bureau du Procureur général au 30 novembre 2010 sur l'application et la situation des individus mis en examen dans le cadre de la loi 975 de 2010

\begin{tabular}{|l|c|}
\hline \multicolumn{1}{|c|}{ Nature des informations traitées } & Nombre de personnes \\
\hline Total des membres des AUC mis en examen & 3046 \\
\hline $\begin{array}{l}\text { Membres des AUC démobilisés collectivement alors qu'ils } \\
\text { étaient en liberté }\end{array}$ & 30 \\
\hline Commandants AUC mis en examen & 893 \\
\hline $\begin{array}{l}\text { Membres des AUC mis en examen et qui au moment de la } \\
\text { démobilisation collective du groupe se trouvaient privés de } \\
\text { liberté }\end{array}$ & 48 \\
\hline $\begin{array}{l}\text { Membres des AUC démobilisés mis en examen individuel- } \\
\text { lement }\end{array}$ & 438 \\
\hline $\begin{array}{l}\text { Total des individus mis en examen, subversion (FARC, ERP, } \\
\text { ELN, EPL, ERG) }\end{array}$ & 4484 \\
\hline Total des mis en examen & \\
\hline
\end{tabular}

Tableau 3: Nombre d'individus mis en examen, incidents de réparation, arrêts de condamnations

\begin{tabular}{|l|c|}
\hline $\begin{array}{l}\text { Individus mis en examen qui ont fait l'objet d'une mise en } \\
\text { accusation }\end{array}$ & 132 \\
\hline $\begin{array}{l}\text { Individus mis en examen ayant terminé la mise en accusation } \\
\text { et attendant la réalisation de l'audience de contrôle de légalité }\end{array}$ & 39 \\
\hline Individus en incident de réparation & 3 \\
\hline $\begin{array}{l}\text { Individus avec condamnations dans le cadre de la procédure } \\
\text { de Justice et de Paix }\end{array}$ & 3 \\
\hline
\end{tabular}

Tableau 4: Types de délits qui se retrouvent dans les procédures de la loi de Justice et de Paix

\begin{tabular}{|l|c|}
\hline \multicolumn{1}{|c|}{ Types pénaux } & Nombre de faits enregistrés \\
\hline Massacres & 1597 \\
\hline Homicides & 45499 \\
\hline Recrutements illégaux & 2144 \\
\hline Disparitions forcées & 4312 \\
\hline Déplacements forcés & 9538 \\
\hline Extorsions & 1777 \\
\hline Enlèvements & 1866 \\
\hline Violence sexuelle & 42 \\
\hline Destruction et appropriation de biens protégés & 124 \\
\hline Torture & 559 \\
\hline
\end{tabular}




\begin{tabular}{|l|c|}
\hline Contrainte illégale & 126 \\
\hline Contributions arbitraires & 1246 \\
\hline Actes de terrorisme & 387 \\
\hline Vols & 2461 \\
\hline Lésions corporelles & 722 \\
\hline Prises d'otages & 27 \\
\hline Trafic, fabrication ou possession de drogues & 68 \\
\hline
\end{tabular}

Tableau 5 : Délits qui ont été avoués

\begin{tabular}{|l|c|}
\hline \multicolumn{1}{|c|}{ Types pénaux } & Nombre de délits avoués \\
\hline Massacres & 20431 \\
\hline Homicides & 1239 \\
\hline Recrutements illégaux & 2045 \\
\hline Disparitions forcées & 6337 \\
\hline Déplacements forcés & 827 \\
\hline Extorsions & 943 \\
\hline Enlèvements & 34 \\
\hline Violence sexuelle & 111 \\
\hline Destruction et appropriation de biens protégés & 432 \\
\hline Torture & 99 \\
\hline Contrainte illégale & 497 \\
\hline Contributions arbitraires & 150 \\
\hline Actes de terrorisme & 1604 \\
\hline Vols & 412 \\
\hline Lésions corporelles & 13 \\
\hline Prises d'otages & 50 \\
\hline Trafic, fabrication ou possession de drogues & \\
\hline
\end{tabular}

Individus condamnés en première instance par les tribunaux de Justice et de Paix: Edwar Cobos Téllez, Jorge Iván Laverde Zapata, Úber Enrique Báez Martínez.

À partir de ces données, quels sont les paramilitaires extradés vers les États-Unis qui continuent à être concernés par la loi de Justice et de Paix? Quels sont ceux qui ont été enlevés? Selon le Bureau du Procureur général, jusqu'au 30 novembre 2010, les paramilitaires impliqués qui ont été extradés font toujours partie du processus établi par la loi.

D’après les résultats obtenus par les statistiques, on peut se permettre de déduire:

- que la procédure judiciaire est insuffisante;

- qu'il y a une forte probabilité pour que la plupart des crimes du paramilitarisme restent impunis; 
- que le droit à la vérité devient plus diffus et lointain, dans la mesure où les enquêtes judiciaires devraient se fonder sur des déclarations qui pourraient être objet à controverse, avec la possibilité que plus de 31000 inculpés soient accueillis et favorisés par le principe d'opportunité;

- que les fins proposées par le gouvernement d'Uribe Vélez ne permettent pas d'atteindre à moyen terme un processus de réconciliation et encore moins de générer les conditions pour la paix dans le pays;

- que les victimes n'obtiennent pas le droit à la vérité, la justice et la réparation;

- que les standards internationaux des droits de l'homme auxquels se soumet la loi ne sont pas remplis. Les conclusions du rapport du haut-commissaire des Nations unies aux droits de l'homme (2010) sont à ce titre éclairantes. Face à la grave crise qui affecte la vie et les droits humains de la population pendant ce conflit armé, il a été amené à recommander un examen approfondi de la loi 975, qui devrait inclure, entre autres choses:

[...] un temps limite pour rendre une déclaration libre, faciliter les mécanismes d'exclusion et étendre la possibilité de rendre des déclarations libres aux démobilisés qui ne sont pas inclus dans la loi. Ainsi, pourraient être consolidées les étapes de la procédure, la possibilité d'accusations collectives, l'obligation d'enquêter et de sanctionner uniquement les crimes graves et de préciser que la réduction de peine commence à partir de la date du jugement. De plus, il serait également souhaitable d'identifier les critères appropriés pour la réparation, d'établir l'obligation de réparer depuis le début des déclarations libres, de renforcer les mécanismes pour que l'État puisse saisir les biens des auteurs des crimes, d'intégrer des approches différentielles et d'assurer une coordination adéquate avec les autres mécanismes de transition ${ }^{33}$.

Ces recommandations aident à repenser le processus de Justice et de Paix, mais il est nécessaire d'aborder l'examen des facteurs structurels qui affectent la coexistence pacifique sur le long terme et qui connaissent une dégradation continue. Pour cette raison, il est nécessaire de promouvoir un dialogue pour que l'établissement de la vérité et de la responsabilité des crimes s'effectue dans un contexte institutionnel. Ainsi, l'État doit s'engager dans une politique qui garantit l'application et le respect des normes du droit international humanitaire pour toutes les parties du conflit.

Un autre aspect directement rattaché à la justice est en lien avec la proposition du gouvernement de Juan Manuel Santos (période 2010-2014) de mettre en œuvre une politique de réparation et de réconciliation intégrale en faveur des victimes. Les premières objections sont issues du Mouvement national des victimes de crimes d'État (MOVICE), relevant que dans le cadre de la loi de Justice et de Paix ne se créent pas les conditions nécessaires pour la réalisation d'un véritable processus de justice qui dégage la vérité et accorde une réparation intégrale aux victimes du conflit armé. En effet, les conditions du processus de négociation et les engagements pris par le gouvernement constituent en elles-mêmes une base permettant de légitimer et de maintenir de nouvelles formes de criminalité, telles que celles incarnées par les bandes émergentes appelées «bandas crimineles» (Bacrim). Les déclarations de certains paramilitaires permettent de mettre en doute la démobilisation de certains fronts et confirment que ce processus ne tend pas à se déplacer vers la justice, la paix et la réconciliation nationale ${ }^{34}$. Selon la CNRR, pour pouvoir rendre possible la réconciliation, il est nécessaire d'organiser un retour digne et durable des personnes déplacées, accompagné par des projets productifs viables et par la restitution des propriétés ${ }^{35}$.

33. Nations unies, «Informe de la Alta Comisionada de las Naciones unidas para los derechos humanos sobre la situación de los derechos humanos en Colombia», Bogota, 30 décembre 2010, $\$ 40$, disponible à l'adresse: http://www.hchr.org.co/documentoseinformes/informes/altocomisionado/informes.php3?cod=14\&cat=11.

34. Movimiento nacional de víctimas de crímenes de lesa humanidad y violaciones a los derechos humanos [Mouvement national des victimes de crimes d'État], «Comunicado público: frente a las políticas del gobierno Santos», $1^{\text {er }}$ février 2011, disponible à l'adresse: http://www.movimientodevictimas.org/pronunciamientos/ comunicados-publicos/item/2185-comunicado-público-frente-a-las-políticas-del-gobierno-santos.html.

35. «La restitución de la tierra. Un derecho inaplazable», Boletín CNRR [publication produite par le CNRR dans le but d'informer le public et surtout les victimes et leurs organisations sur les mesures prises par la Commission dans le cadre de la loi 975 de Justice et de Paix], Bogota, ${ }^{\circ}{ }_{15}$, octobre 2010. 
En guise de conclusion, dans le cadre des discussions menées par l'équipe de recherche avec certains dirigeants d'organisations sociales et de mouvements politiques, sont présentés des éléments qui facilitent la lecture contextuelle au regard des standards internationaux et de l'indépendance judiciaire.

L'analyse des sources de cette recherche nous oblige à citer à nouveau l'expérience vécue à San José de Apartadó, qui a fait l'objet d'une recherche antérieure développée avec le soutien de l'Université autonome de Colombie ${ }^{36}$, où ont été pointées les difficultés rencontrées pour accéder et obtenir justice, le manque de respect et de protection pour les communautés touchées par le conflit et la consolidation du modèle économique du paramilitarisme dans la région d'Urabá par rapport aux standards du droit international humanitaire, comme le constate la Cour constitutionnelle dans son arrêt T-249/03 ${ }^{37}$, qui explique les efforts et la lutte nécessaires pour ne pas laisser les crimes contre l'humanité impunis.

Nous pouvons encore aujourd'hui nous interroger sur les points suivants: quand allons-nous connaître la vérité de l'Urabá ${ }^{8}$ et les responsables de crimes contre l'humanité? Que s'est-il passé au cours des enquêtes sur le général Rito Alejo del Río ${ }^{39}$ ? Quand sera explicité le rôle directeur de l'ex-président Álvaro Uribe dans la stratégie mise en œuvre en Antioquia dans les années 1995-1997 avec la création des CONVIVIR institutionnalisant le paramilitarisme ${ }^{40}$ ?

Au sein du Congrès de la République de Colombie, les sénateurs et les représentants du parti politique d'opposition Polo democratico alternativo (Pôle démocratique alternatif) ont dénoncé publiquement la grave infiltration du paramilitarisme ${ }^{41}$, en espérant que l'impunité ne soit pas un principe directeur lors de l'émission de nouvelles normes juridiques qui pourraient bénéficier au crime parapublic.

Le processus vécu en Colombie n'est pas étranger à d'autres qui se sont développés dans différentes parties du monde, avec le travail de commissions de vérité et de récupération de la mémoire historique, ce qui nous a conduit à un dialogue avec des hommes et des femmes d'autres régions et latitudes qui ont vu venir la mort dans leurs maisons, dans leurs communautés, et qui connaissent également de complexes réalités économiques, sociales, religieuses et politiques. Les expériences vécues en Afrique du Sud, au Rwanda, en Bosnie, en Palestine, au Guatemala, au Salvador, au Nicaragua, en Argentine, au Chili, au Pérou, au Libéria, en Inde, en Irak, au Kosovo, en Afghanistan et au Chiapas, entre autres $^{42}$, ont eu leur propre contexte, mais ces pays s'identifient les uns aux autres dans

36. G. Cuartas Montoya, Ante la impunidad y la indiferencia, Bogota, Fondo de publicaciones Universidad autónoma de Colombia, 2007.

37. Arrêt T-249/03, enregistrement T-668169: action de tutelle instaurée par Javier Giraldo Moreno contre le Procureur général de la nation; magistrat: $\mathrm{D}^{\mathrm{r}}$ Eduardo Montealegre Lynett.

38. L'Urabá est une sous-région géographique de la Colombie qui a été longtemps victime de la violence paramilitaire, et qui continue à être un champ d'action pour les bandes criminelles. Dans cette zone, les autodéfenses ont effectué une série d'actions violentes telles que la prise en otagedes syndicalistes, des paysans, des gauchistes, des défenseurs des droits de l'homme et des habitants de la région, qui ont été torturés et tués, car soupçonnés d'être membres ou sympathisants de la guérilla.

39. Cet ancien général de l'armée nationale est sans aucun doute l'un des militaires colombiens les plus controversés et contestés en raison de ses liens avec les paramilitaires qui ont commencé à partir du début des années 1990, lorsqu'il était chef de la Brigade XVII dans l'Urabá. C'est à cette époque qu'a débuté l'assaut sanglant des paramilitaires, qui a généré des dizaines de plaintes dénonçant la collaboration entre les troupes du général Rito Alejo del Río et les paramilitaires. Finalement, en août de l'année dernière, un juge a condamné le général Rito Alejo del Río à 25 ans de prison pour l’un des meurtres commis par des paramilitaires dans l'Urabá en 1997.

40. Les CONVIVIR ont été créés par le décret 356 du 11 février 1994 en tant que coopératives pour la prestation de services de surveillance et de sécurité privée. Avec le processus de Justice et de Paix, de nombreux CONVIVIR dans les régions d'Antioquia, la côte Caraibe et les plaines orientales ont été créés par des trafiquants de drogue ou par des paramilitaires, pour s'approvisionner légalement en armes, se financer et protéger leurs groupes avec des licences légales. Selon le Parquet, dans la région d'Antioquia ont été constitués au moins 87 de ces organisations, dont 13 opéraient dans l'Urabá. C’est pendant la période d'Álvaro Uribe Vélez comme préfet d'Antioquia, entre 1995 et 1997, qu'ont été créés la majorité des CONVIVIR dans cette région du pays.

41. Voir «Se comprueba infiltración paramilitar en Congreso colombiano», Colombia Hoy, 11 octobre 2006, disponible à l'adresse: http://blog.colombiahoy.org/2006/11/10/se-destapa-la-infiltracin-paramilitar-en-elcongreso-de-colombia.aspx.

42. Voir H. Zin, B. Matias, "El mundo en guerra: todos los conflictos bélicos que siguen activos», 20 minutos, 13 septembre 2008 , disponible à l'adresse: http://www.webislam.com/?idt=10935. 
les graves violations commises contre la population civile, avec la formation de groupes d'autodéfense de l'État qui exécutent les atrocités que les forces gouvernementales ne peuvent pas effectuer, lorsqu'il ne s'agit pas de dictatures militaires ou d'interventions étrangères consolidées dans le sang et le feu.

Le rôle des organisations de la société civile, à travers la résistance civile pour la défense de la paix, a été renforcé par la création de réseaux de solidarité et de soutien mutuel que nous devons identifier et adapter sans pour autant copier les modèles de négociation de paix. Notre réalité, comme celle de chaque pays, est concrète et mérite une construction collective pour répondre spécifiquement à la situation politique et économique propre, sans perdre de vue le rôle du gouvernement dans la promotion de la guerre.

Au milieu de l'une des plus complexes violations des droits humains des hommes et des femmes au nom de la sécurité démocratique, se révèle la fragilité de la démocratie limitée. Cette réalité est connue de la communauté internationale, particulièrement de l'Union européenne ${ }^{43}$. Nous assistons à un scandale considérable, qui a abouti à la réélection d'un président qui a obtenu une grande partie de son «triomphe» dans des votes soutenus par le narcotrafic et le paramilitarisme présents dans 712 municipalités au cours de la période 1994-2004 (au moins 480 maires, 1200 conseillers et 1200 hommes d'affaires ayant des liens étroits avec le paramilitarisme; et la participation d'officiers et de sous-officiers de la force publique) ${ }^{44}$. En outre, la Colombie est confrontée à une crise appelée "parapolitique » ${ }^{45}$, dans laquelle le gouvernement d'Uribe a assumé l'engagement de légaliser et de légitimer les structures criminelles. Étant soutenu par l'argent de ces structures qui lui ont permis d'arriver à un pouvoir illégitime, son gouvernement a engendré une corruption gangrenant les différentes institutions de l'État.

En Colombie s'est développée une législation suffisante pour légaliser un processus légitimant des dispositifs de mort et des violations des droits de l'homme, même si les États-Unis «certifient» ${ }^{6}$ que cette politique est une protection de la démocratie. Les milliers de personnes assassinées ou disparues, la réalité du déplacement forcé ou la perte de terres de milliers de personnes, sont désormais «cachés» devant la gravité du trafic de drogue, les groupes paramilitaires et les groupes de contrôle et de discipline sociale.

Dans ce cadre, les mouvements et les organisations sociales exigent une série d'ajustements au processus de Justice et de Paix, élaboré par le gouvernement, en partant d'une solution pacifique au conflit social et armé, à savoir :

- Dans le processus de démobilisation l'État a légitimé le paramilitarisme en le liant à la force publique et / ou aux services de sécurité, ce que l'on nomme désormais «bandas crimineles» (Bacrim). Certains parlent de processus différents, d'autres affirment que c'est la poursuite et la consolidation de la structure de contrôle et de l'usage des terres.

- Pas de pardon et pas d'oubli. Non à toutes les lois d'amnistie générale («Punto final»), de l'obéissance due, d'auto-amnistie ou de toute autre mesure qui pourrait laisser les crimes dans l'impunité.

- Réaliser en Colombie, ouvertement et avec une totale transparence, les processus de démobilisation et de soumission à la justice des groupes paramilitaires, en informant l'opinion publique nationale et internationale des résultats de ceux-ci, pour que les organisations sociales, la communauté, les forces politiques et, en particulier, les victimes, puissent avoir toutes les garanties pour exprimer leurs opinions et propositions de mémoire, vérité, justice et réparation intégrale.

- Enquêter sur les crimes de guerre et crimes contre l'humanité commis par les groupes paramilitaires et punir les auteurs intellectuels et matériels de ceux-ci.

43. Voir A. Parada Fernández, Ironía histórica cínica, Bogota, Fondo de publicaciones Universidad autónoma de Colombia, 2004, p. 386-398.

44. Voir Fundación Seguridad y Democracia, «La magnitud de la verdad del paramilitarismo», disponible à l'adresse: http://www.erta-tcrg.org/cri6224/2008/paramilitaires/pdf/verdadSuMagnitud.pdf.

45. Nom employé pour évoquer le scandale politique qui a éclaté en Colombie à partir de 2006 lors de la divulgation des liens politiques avec les paramilitaires, après la démobilisation de plusieurs groupes qui formaient les AUC.

46. Ejército de Colombia [Armée de Colombie], «EEUU certifica a Colombia en derechos humanos», 2010, disponible à l'adresse: http://www.ejercito.mil.co/?idcategoria=263410. 
- Dévoiler les noms des bailleurs de fonds, les complices et les bénéficiaires de la structure paramilitaire et les méthodes et les mécanismes d'impunité mis en œuvre jusqu'à aujourd'hui. De même, établir clairement ceux qui ont été les victimes, et les raisons pour lesquelles ils ont été éliminés. Cet éclairage devrait être fait à partir de l'année 1945, date à laquelle a débuté le processus d'extermination des mouvements populaires (génocide contre le mouvement Gaitanista ${ }^{47}$ ).

- L'État, en tant qu'institution, doit reconnaître le génocide de l'UP et assumer la responsabilité qui correspond à l'anéantissement de ses membres, qui souhaitaient une action politique pour la paix et qui ont été trahis et exécutés par le paramilitarisme avec l'alliance illicite des forces de l'État ${ }^{48}$.

- L'État doit indemniser intégralement les victimes du paramilitarisme, en accordant une réparation patrimoniale pour les dommages moraux, matériels, sociaux, politiques et psychologiques causés.

- Le gouvernement colombien doit prêter attention aux recommandations d'organismes internationaux comme l'ONU et l'OEA, et d'institutions nationales comme le «Défenseur du Peuple» (Ministère public), concernant la mauvaise politique de soi-disant «dialogue» avec les groupes paramilitaires.

- Clarifier publiquement le destin des terres et autres biens que les groupes paramilitaires ont confisqué aux milliers de paysans et aux autres personnes aujourd'hui déplacées et marginalisées dans les centres urbains. Il suffit de rappeler la «pacification de l'Urabá», ce processus qui est resté dans l'impunité et qui se poursuit encore aujourd'hui avec le harcèlement des communautés qui ont résisté à la politique paramilitaire dans la région.

47. Le mouvement Gaitanista fait référence à un mouvement dirigé par l'homme politique Jorge Eliécer Gaitán. Candidat du parti libéral à la présidence de la République pour la période 1946-1950, il a été assassiné le 9 avril 1948 à Bogota, fait qui a généré d'énormes manifestations connues sous le nom de «Bogotazo». Cette période 1948-1960, connue comme "la période de la violence», a commencé à la suite de l'assassinat du leader politique, et a été marquée par le terrorisme, les meurtres et les destructions de biens. Les meurtres prémédités, massifs et systématiques de l'État envers des membres du mouvement Gaitanista dans les années 1940 et 1950, ainsi que l'assassinat de son chef, Jorge Eliécer Gaitán, constituent l'un des cas les plus documentés et dramatiques de génocide politique de l'histoire de la Colombie.

48. Voir O. Palacios, I. David, El genocidio político contra la unión patriótica, Bogota, Universidad nacional de Colombia, 2009. 